\title{
Investigations of Thermocouple Drift Irregularity Impact on Error of their Inhomogeneity Correction
}

\author{
Su Jun ${ }^{1}$, Orest Kochan ${ }^{2}$ \\ ${ }^{1}$ School of Computer Science, Hubei University of Technology, Hubei, China, sjhosix@gmail.com \\ ${ }^{2}$ Department of Metal Physics, Ivan Franko National University of L'viv, 1, Universytetska str., 79000, Lviv, Ukraine, \\ oko@tneu.edu.ua
}

\begin{abstract}
The article examines: (i) the reasons of error due to thermoelectric inhomogeneity of electrodes of thermocouples acquired during prolonged use; (ii) the neural network method of error correction based on a generalization of verification results in several temperature fields; (iii) the method of investigating the impact of changing the speed of the conversion characteristic drift of thermocouple on error correction; (iv) results of this investigation. It is shown that residual error for type $\mathrm{K}$ thermocouples at the $5 \%$ level of significance does not exceed $\mu \pm 0.46^{\circ} \mathrm{C}$ and one at the $10 \%$ level of significance does not exceed $\pm 0.25{ }^{\circ} \mathrm{C}$.
\end{abstract}

Keywords: Temperature measurement errors, thermocouple, error due to inhomogeneity.

\section{INTRODUCTION}

$\mathrm{T}$ HERMOCOUPLES (TC) are the most widely used temperature sensors [1] in the range $500-1500^{\circ} \mathrm{C}$ in industry, despite their considerable drawbacks. That is why sensors with different physical background have been studied. There are Distributed temperature sensing systems, laser sensors, and thermal noise thermometer [2] sensors. But these types of sensors are still less precise and less safe than thermocouples. The main drawback of thermocouples is their relatively high error, which dominates in measurement channel. Therefore, error of sensor is the biggest error among errors of other components of measurement channel [3-5]. The same situation is typical for other sensors $[6,7]$.This fact calls for research of effective error decreasing methods.

General temperature measurement error of thermocouples can be split into a few components. For most of them the reliable methods of error correction are known. Like for the following components: (i) initial deviation of conversion characteristics (CC) of thermocouples from nominal [7]; (ii) significant deviation of $\mathrm{CC}$ during operation, which is connected with degradation of electrodes of thermocouples under influence of high temperature and operation time [5, 9-11]; (iii) error caused by cold junction effect, and so on [1, $2,12]$.

But the most critical error is error caused by inhomogeneity of thermoelectrodes of thermocouple. This inhomogeneity is acquired during operation under influence of time and high temperature. Inhomogeneity has been considered to be a problem for a long time [13, 14]. But modern authors argue inhomogeneity is still a serious problem $[15,16]$, and it is considered to be the main reason of thermocouple error [17]. Estimated value of inhomogeneity error according to [16] is about $5-10^{\circ} \mathrm{C}$ for type K thermocouples.

For homogeneous thermocouple, output electromotive force (e.m.f.) is functionally dependent on the temperature difference between hot and cold junctions. But for an inhomogeneous thermocouple, which was used in operating conditions, e.m.f. depends on the profile of temperature field as well. Thus, change of temperature field along thermoelectrodes leads to changes in output signal even if the temperatures of hot and cold junctions remain the same. This inhomogeneity error led to the formulation of the hypothesis [13] that it is impossible to decrease the error of thermocouple. Let us consider the impact mechanism of the error due to inhomogeneity for developing a method of its correction.

Impact mechanism of thermocouple electrode inhomogeneity acquired on a generated thermo-e.m.f is explained in Fig.1. [18]. There is a thermocouple chromelalumel in Fig.1.a), that consists of chromel and alumel electrodes. This thermocouple is utilized in thermal field of an object which is defined by the line ABCD. Thermoelectrodes deteriorated due to oxidation of their components, diffusion of admixtures, recrystallization, etc. [14] during their usage time $\tau$. Therefore, CC of section, i.e. $l_{i}$ deviates from the nominal. The deviation of thermocouple CC from the nominal $\Delta T$ for time $\tau$ and a given temperature $T_{i}$ that is applied to the section $l_{i}$ is shown in Figs.1.b) and 1.c) [19].

If a profile of a temperature field that is observed along the thermoelectrodes has been changed (new value is AB1C1D), then the section $l_{i}$ moves from the temperature of a regular operation $T_{i}$ to the temperature $T_{i+1}$. Thus, deviation of CC of both thermoelectrodes from the nominal will be changed (within this section $l_{i}$ ). This deviation will change from the old value of the temperature $T_{i}$ to the new one $T_{i+1}$ (shown with the arrows in Figs.1.b) and 1.c)). Similar changes will be observed for other sections of thermocouples that are found in the area of temperature gradient. Therefore, thermo-e.m.f that is generated by thermocouple will change, though temperatures of the hot and cold junctions will remain unchanged (Fig.1.a)). Thus, an error of temperature measurement from thermoelectric inhomogeneity of electrodes of thermocouple appears. The mentioned feature 
of old thermocouples (that were used for a long time at high temperature) led to the conclusion that thermocouple errors cannot be corrected.

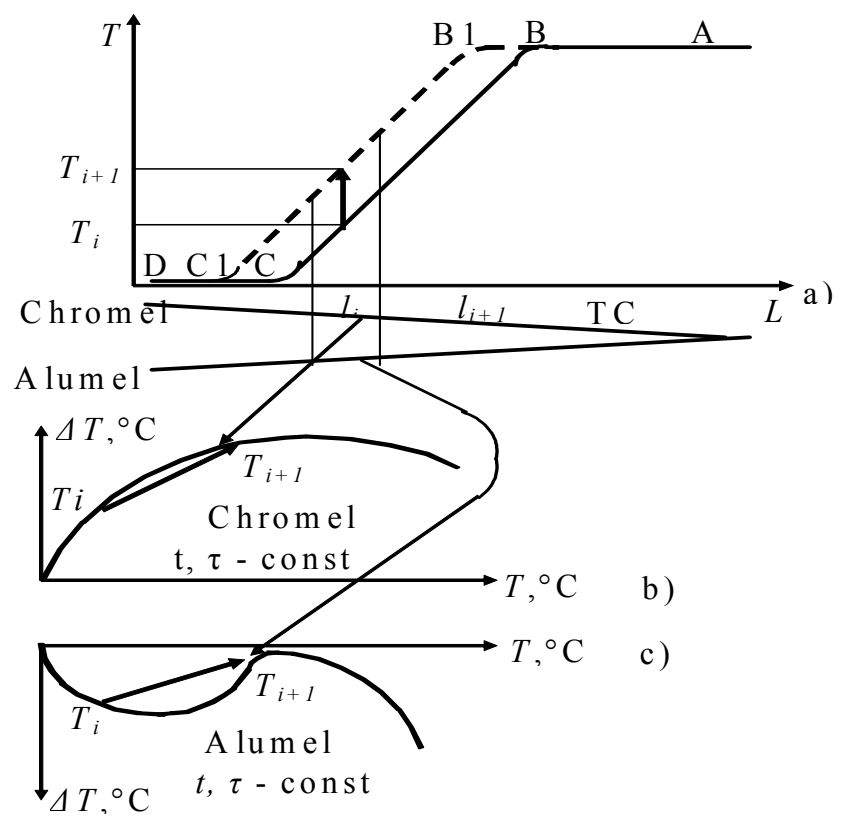

Fig.1. Creation of an error obtained in the process of continuous exploitation of the thermoelectric irregularity in thermocouple electrodes.

All attempts for decreasing the error of acquired inhomogeneity $[20,21]$ showed that correction of drift of $\mathrm{CC}$ should be done for each segment of thermoelectrode separately. Such correction can be performed by drift prediction for each segment using individual mathematical model. Unfortunately, the continuous individual prediction of $\mathrm{CC}$ drift of segment is not reliable. High accuracy of prediction can be achieved by its periodic correction using the determined values of real $\mathrm{CC}$ drift from the nominal.

Meanwhile, verification or calibration of thermocouple allows us to obtain a total error for all segments of TC. This total error should be distributed between all segments. There were proposed four possible empirical criteria for error distribution among drift models for separate segments [22]. But these criteria have never been proved experimentally. Indeed, incorrect criteria may increase impact on temperature measurements caused by error of acquired inhomogeneity.

Detailed analysis of error of acquired heterogeneity features was performed in [23]. Such analysis enabled the development of methods for error correction of acquired inhomogeneity and its compensation [24, 25]. Method for correction of this error was proposed in [24]. It is based on experimental research of thermocouple error. These experiments are performed during the periodical verification of thermocouple that is located in different temperature fields.

Main goal of this paper is to research error of method for acquired thermoelectric inhomogeneity of thermocouple error correction [24] that is caused by specific variation of $\mathrm{CC}$ drift of segments of the electrode process.

\section{METHOD FOR CORRECTION OF THE ERROR OF ACQUIRED THERMOELECTRIC HETEROGENEITY OF THERMOCOUPLES}

Assume the total prediction error of a drift (that is evaluated experimentally) is distributed among the segments of thermocouple correctly. Afterwards, a correction of individual mathematical models of $\mathrm{CC}$ drift for all segments of the thermocouples is provided. Thus, prediction error for other profiles of temperature fields converges to zero. This principle was used for the proposed method [24].

First implementation of the proposed method requires the following operations:

1. splitting each thermoelectrode into $N$ segments equal in length;

2. $\mathrm{CC}$ of each segment is considered to be constant in length;

3 . verification of $\mathrm{TC}$ into $2 \mathrm{~N}$ profiles of temperature field;

4. solving the following system of equations.

$$
\left\{\begin{array}{l}
k_{1}^{\mathrm{X}} \Delta_{1-1}^{\mathrm{X}}+k_{2}^{\mathrm{X}} \Delta_{1-2}^{\mathrm{X}}+\ldots+k_{\mathrm{N}}^{\mathrm{X}} \Delta_{1-\mathrm{N}}^{\mathrm{X}}+k_{1}^{\mathrm{A}} \Delta_{1-1}^{\mathrm{A}}+ \\
+k_{2}^{\mathrm{A}} \Delta_{1-2}^{\mathrm{A}}+\ldots+k_{\mathrm{N}}^{\mathrm{A}} \Delta_{1-\mathrm{N}}^{\mathrm{A}}=\Delta_{1}^{\mathrm{D}} \\
k_{1}^{\mathrm{X}} \Delta_{2-1}^{\mathrm{X}}+k_{2}^{\mathrm{X}} \Delta_{2-2}^{\mathrm{X}}+\ldots+k_{\mathrm{N}}^{\mathrm{X}} \Delta_{2-\mathrm{N}}^{\mathrm{X}}+k_{1}^{\mathrm{A}} \Delta_{2-1}^{\mathrm{A}}+ \\
+k_{2}^{\mathrm{A}} \Delta_{2-2}^{\mathrm{A}}+\ldots+k_{\mathrm{N}}^{\mathrm{A}} \Delta_{2-\mathrm{N}}^{\mathrm{A}}=\Delta_{2}^{\mathrm{D}} \\
\ldots \ldots \ldots \ldots \ldots \ldots \ldots \ldots \ldots \ldots \ldots \ldots \ldots \ldots \ldots \ldots \\
\cdots \ldots \ldots \\
k_{1}^{\mathrm{X}} \Delta_{2 \mathrm{~N}-1}^{\mathrm{X}}+k_{2}^{\mathrm{X}} \Delta_{2 \mathrm{~N}-2}^{\mathrm{X}}+\ldots+k_{\mathrm{N}}^{\mathrm{X}} \Delta_{2 \mathrm{~N}-\mathrm{N}}^{\mathrm{X}}+k_{1}^{\mathrm{A}} \Delta_{2 \mathrm{~N}-1}^{\mathrm{A}}+ \\
+k_{2}^{\mathrm{A}} \Delta_{2 \mathrm{~N}-2}^{\mathrm{A}}+\ldots+k_{\mathrm{N}}^{\mathrm{A}} \Delta_{2 \mathrm{~N}-\mathrm{N}}^{\mathrm{A}}=\Delta_{2 \mathrm{~N}}^{\mathrm{D}}
\end{array}\right.
$$

where $\Delta_{1-1}^{X} \quad \ldots \quad \Delta_{1-N}^{X} \quad-$ prediction for individual mathematical models of CC drift for segments of chromel from 1 to $N$ in the first profile of temperature field of verification (the first subscript defines the number of temperature field; the subscript after the dash defines the number of a segment); $\Delta_{2-1}^{\mathrm{X}} \ldots \Delta_{2-\mathrm{N}}^{\mathrm{X}} \ldots \Delta_{2 \mathrm{~N}-1}^{\mathrm{X}} \ldots \Delta_{2 \mathrm{~N}-\mathrm{N}}^{\mathrm{X}}-$ prediction for individual mathematical models of $\mathrm{CC}$ drift for segments of chromel from 1 to $N$ in profiles $2-2 \mathrm{~N}$ of temperature field of verification; $\Delta_{1-1}^{A} \quad \ldots \quad \Delta_{1-N}^{A} \quad-$ prediction for individual mathematical models of CC drift for segments of alumel from 1 to $N$ in the first profile of temperature field of verification; $\Delta_{2-1}^{\mathrm{A}} \ldots \Delta_{2-\mathrm{N}}^{\mathrm{A}} \ldots \Delta_{2 \mathrm{~N}-1}^{\mathrm{A}} \ldots \Delta_{2 \mathrm{~N}-\mathrm{N}}^{\mathrm{A}}-$ prediction for individual mathematical models of $\mathrm{CC}$ drift for segments of alumel from 1 to $N$ in profiles $2-2 \mathrm{~N}$ of temperature field of verification; $\Delta_{1}^{D} \ldots \Delta_{2 \mathrm{~N}}^{D}$ - real deviation of TC CC from nominal in profiles $1-2 \mathrm{~N}$ of temperature field of verification.

If the amount of profiles for verifying temperature field equals to the amount of segments that form both electrodes, then set (1) has the unique solution. Equalizing coefficients $k_{1}^{\mathrm{X}} \ldots k_{\mathrm{N}}^{\mathrm{X}}$ for all chromel segments and $k_{1}^{\mathrm{A}} \ldots k_{\mathrm{N}}^{\mathrm{A}}$ for all alumel segments can be evaluated unambiguously. These coefficients will decrease prediction error for individual 
mathematical models of $\mathrm{CC}$ drift for the segments of both electrodes. This will lead to improving prediction accuracy in general and, therefore, to improving accuracy of temperature measurements.

Main disadvantage of the described method is high labor coefficient of such periodical verification. Also the collision appears. There is a condition that within one segment CC drift is the same, therefore, we can use this fact as a criterion for picking up segments. Thus, the amount of segments $N$ should be increased for improving prediction accuracy. Then, individual mathematical models of segment drift can describe more precisely a trend of drift of CC of segments. But with the increasing amount of sections, the amount of profiles for verifying temperature field rises faster, so the labor coefficient is increasing as well.

The labor coefficient decreasing can be reached by using artificial neural networks [24]. Training set for neural network (NN) is created in the following way. Each vector consists of TC verification results obtained for one profile of the temperature field. Input neurons of $\mathrm{NN}$ accept measured temperature values for all the sections of thermocouple (for the given profile of the temperature field). Link between segment number and number of input neuron should be kept. Output of $\mathrm{NN}$ is absolute error of thermocouple that is obtained during the verification in this profile of temperature field. This error equals to deviation of total error prediction for all segments of thermocouple from its real error (for the given profile of the temperature field).

NN-based correction method provides labor coefficient decreasing a few times. But it has some shortcuts. Its error depends on $\mathrm{CC}$ drift heterogeneity of thermoelectrode segments. Therefore, a residual error of the proposed method should be analyzed. This error is caused by individual heterogeneity of the $\mathrm{CC}$ drift process at each segment of electrodes of thermocouple.

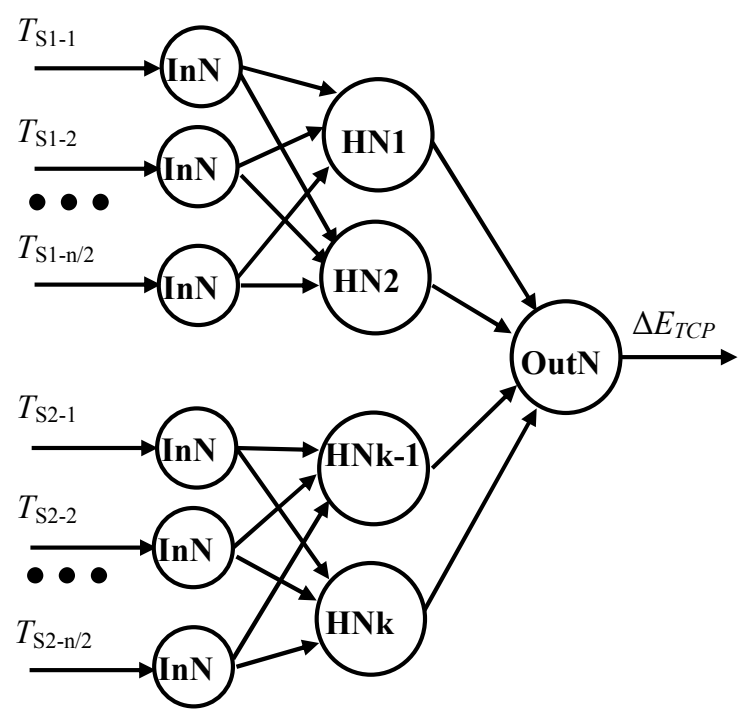

Fig.2. Structure of neural network.

Structure of the NN for CC drift of thermocouple decomposition is given in Fig.2. NN (a three layer perceptron) contains an input layer (distributive neurons), hidden layer (sigmoid activation function) and one output linear neuron. Number of input neurons is equal to number of segments of a thermocouple. Number of hidden layer neurons is less than half the number of verification results, otherwise perceptron will become associated memory without generalization properties. Temperatures of corresponding segments are sent to the inputs of NN.

\section{METHOD FOR FOR RESEARCH OF THE NO EXCLUDED ERROR OF THE PROPOSED APPROACH}

Experimental research of the proposed method does not allow investigating completely its metrological characteristics. Such investigations are strictly limited and do not exhaust possible individual features of errors of thermocouples and impact of their operating conditions. Analytical research of error of the method requires development of a set of mathematical models for individual drift of $\mathrm{CC}$ of segments of thermoelectrodes. Thus, the optimal method for the research of metrological characteristics of the proposed method is simulation modeling.

Training set for neural network is developed based on the results [16] of error evaluation for chromel and alumel with the electrodes' diameter of $1.2 \mathrm{~mm}$ and during the 1000-hour operational time. These results are based on approximation results of experimental research [19]. Each electrode consists of 24 segments. Extreme segments (that are located at the temperatures of hot and cold junctions, respectively) did not change their temperature. Eight segments from the rest were located in the gradient area of temperature field. Changes of temperature field profile were done by parallel shift of temperature field profile on $50{ }^{\circ} \mathrm{C}$ (change of insertion depth is the most critical usage mode for a heterogeneous thermocouple). Such change corresponds to verification in 28 profiles of temperature field.

Each segment has its own variation of CC drift that has been simulated by the sinusoid. This sinusoid has amplitude, frequency and phase that change randomly. This mathematical model can be described as

$$
U_{N R}=r 1 \cdot A \sin \left(\left(\omega_{M I N}+r 2 \cdot \Delta_{\omega}\right)+r 3 \cdot 2 \pi\right)
$$

where $U_{N R}$ - current value of thermo-e.m.f drift variation for random segment; $A, \omega_{M I N}, r 3 \cdot 2 \pi$ - amplitude, sinusoidal initial frequency and phase, that simulates drift variation for random segment; $r 1, r 2, r 3$ - random coefficients, that are generated according to normal distribution law within the range from zero to one, $r \in[0 ; 1]$

Maximum training set consists of 28 lines. Each row of the training set simulates an evaluation result for one profile of temperature field along the thermoelectrodes. In order to decrease the verification labor coefficient of thermocouple, we researched error change dependence while training sets were reduced to 21,14 and 7 rows. Decreasing the amount of rows of the training set is performed in the same way - by deleting forth row, third and fourth rows or second, third and fourth rows out of each four rows. This approach 
simulates decreasing the amount of verifications four times. Testing set always remains constant. It always contains the maximum training set ( 28 rows). Thus, the testing set is used for research error of missed verifications.

Three-layer perceptron is used as neural network [26]. Its input neurons perform only resending functions. Amount of such neurons equals to total amount of segments of both thermoelectrodes - 48. Main generalizing features have neurons of the hidden layer. Sigmoid was used as an activation function. Amount of neurons for the hidden layer was determined experimentally, and equals to 3 . This amount is less than half from the amount of temperature field profiles that were used for verification. Therefore, there is no danger for the perceptron to use its generalization features. Output neuron is linear, and its activation function is 1 . Artificial neural network was trained according to the back-propagation algorithm using the Levenberg-Marquardt method. Maximum epochs for training - 4000 (training time is about 4 minutes).

\section{RESEARCH RESULTS}

The initial temperature field profile along thermoelectrodes of inhomogeneous thermocouple was formed as follows:

- Zones from 1 to 8 - are at zero temperature (no drift of conversional characteristic (CC));

- Zones from 9 to 16 - are at $100{ }^{\circ} \mathrm{C}$ temperature gradient for each area (drift of CC according [19]);

- Zones from 17 to 24 - are at temperature $800{ }^{\circ} \mathrm{C}$ (drift of CC according [19]).

Change of temperature field profile during experimental research modeling of inhomogeneous thermocouple is done stepwise by $50{ }^{\circ} \mathrm{C}$. Then we get 28 temperature field profiles for verification (both zones 1 and 24 remain at initial temperatures). The steps for temperature field profile change lay along the $\mathrm{X}$-axis. The ratio of non-excluded error of error correction from temperature field change along thermoelectrodes to maximum value of this error $\Delta_{1}^{\mathrm{D}} \ldots$ $\Delta_{2 \mathrm{~N}}^{\mathrm{D}}$ for current conditions lay along the Y-axis.

Fig.3. shows non-excluded error of error correction dependence from acquired inhomogeneity from the number of verifications at amplitude of individual error irregularity of acquired inhomogeneity rate of $5 \%$ from its maximum value. As can be seen from Fig.2., the average value of error correction is small, when maximum error is fairly high and relatively weakly depends on the number of verifications.

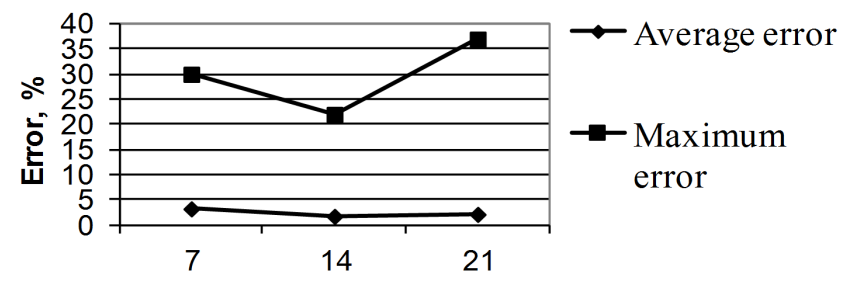

Number of verifications

Fig.3. Non-excluded errors of error correction dependence from acquired inhomogeneity from the number of verifications for rate at $5 \%$ drift maximal irregularity.
Fig.4. shows the individual implementation of nonexcluded error of error correction dependence from acquired inhomogeneity for seven verifications. As it can be seen from Fig.4., the amplitude of most errors does not exceed $10 \%$, maximum deviation is located near the edges of the graph, which correspond to a large change of temperature field profile.

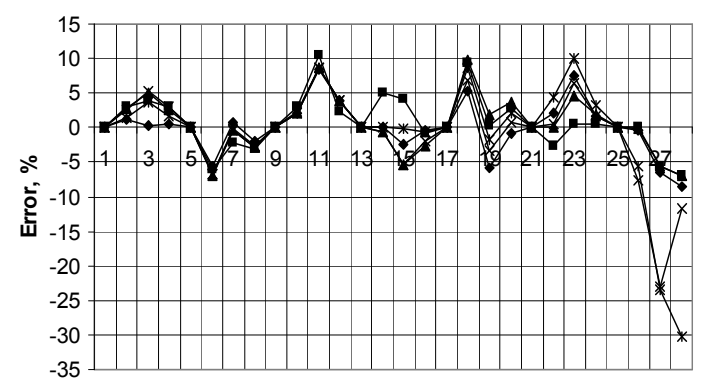

Number of zone

Fig.4. Non-excluded correction error of inhomogeneity for rate at $5 \%$ drift maximum irregularity for 7 verifications (average error is $3.2 \%)$.

Fig.5. shows non-excluded error of error correction dependence of acquired inhomogeneity from the number of verifications during individual error irregularity amplitude of acquired inhomogeneity rate at $10 \%$ from its maximum value. As graph on Fig.5. shows, an average error value is minor too, while maximum error is pretty large and proportional to the maximum number of verifications (although it is possible to expect an inverse dependence).

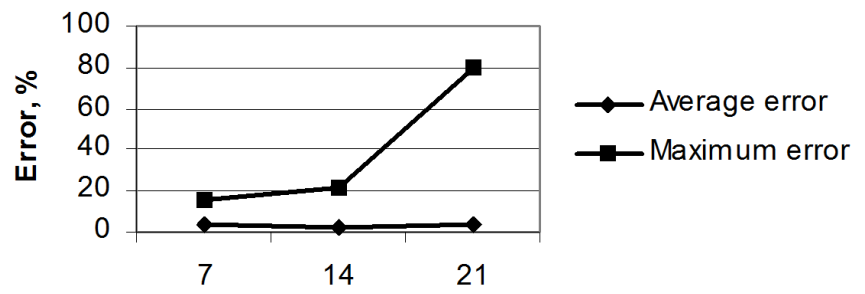

Number of verifications

Fig.5. Non-excluded error of correction error dependence of acquired inhomogeneity from number of verifications for rate at $10 \%$ drift speed maximum irregularity.

As can be seen from Fig.6., which represents individual implementation for error of error correction dependence from acquired inhomogeneity for seven verifications, maximum error does not exceed $15 \%$ and is distributed along all temperature field profiles.

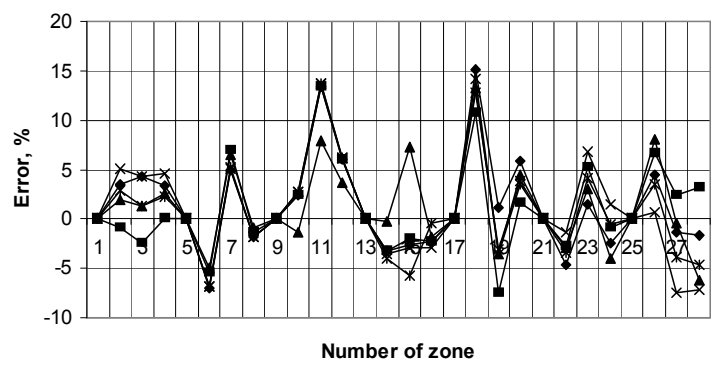

Fig.6. Non-excluded correction error of inhomogeneity rate at $10 \%$ drift speed maximum irregularity for 7 verifications (average error is $3.3 \%$, maximal is $15 \%$ ). 
Fig.7. shows error of correction error dependence of acquired inhomogeneity from the number of verifications for individual error irregularity amplitude from acquired inhomogeneity rate at $15 \%$ from its maximum value.

As can be seen in Fig.7., an average correction error value is still minor (less than $5 \%$ ) and practically does not depend on the number of verifications. Maximum error is relatively small too and remains almost constant.

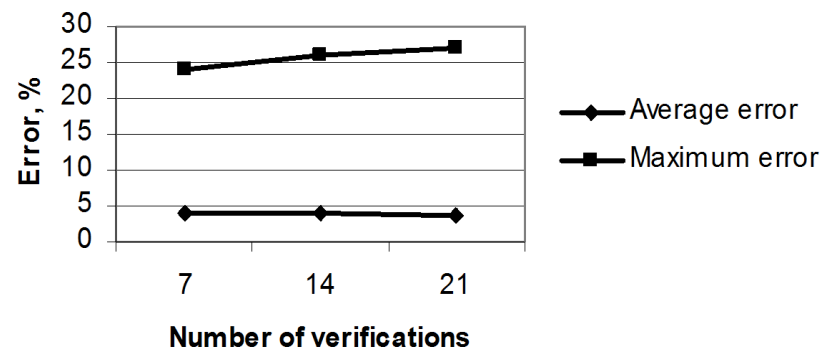

Fig.7. Non-excluded error of error correction dependence of acquired inhomogeneity from number of verifications for rate at $15 \%$ drifts maximum irregularity.

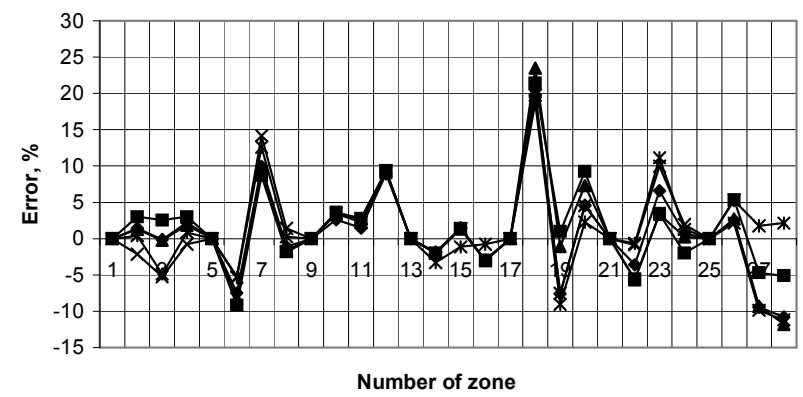

Fig.8. Non-excluded correction error of inhomogeneity rate at $15 \%$ drift speed maximum irregularity for 7 verifications (average error is $3.9 \%$, maximal error $24 \%$ ).

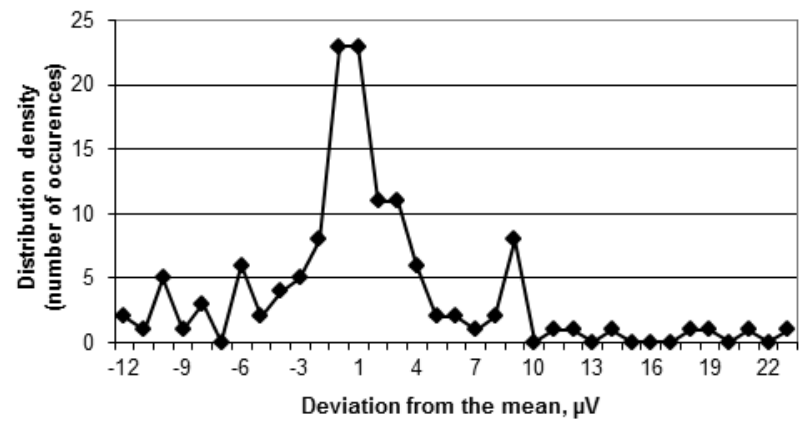

Fig.9. Distribution of absolute non-excluded error of correction error for individual drift irregularity.

However, as can be seen from Fig.8., which represents individual implementation for error of error correction dependence from acquired inhomogeneity for seven verifications, maximum error does not obviously correspond to the maximum change of the temperature field profile.

Fig.9. shows distribution of non-excluded error of error correction of acquired thermocouple inhomogeneity for rate up to $15 \%$ of maximum drift irregularity and during reducing the number of temperature field profiles to seven (for the most complex case). As Fig.9. shows, correction error distribution law can be considered close to normal. Peaks at $9 \mu \mathrm{V},-6 \mu \mathrm{V}$ and $-10 \mu \mathrm{V}$ cannot be explained by limited sample size. They are probably caused by features of NN training analogically to peak at zone 28 in Fig. 4.

Therefore, probability of values calculated by neural network with large deviation from verification results is quite low.

Standard deviation $\sigma$ for single implementation can be defined according to the formula [22]:

$$
\sigma=\sqrt{\frac{1}{n} \sum_{i=1}^{n}\left(X_{i}-M\right)^{2}} \approx 6 \mu \mathrm{V}
$$

where $X_{i}-M$ - absolute error of error correction values from acquired inhomogeneity.

Calculations have shown that the estimation of nonexcluded correction error, defined within $\pm 3 \sigma$ range, is $\pm 18.5 \mu \mathrm{V}$. Thus, $95 \%$ of non-excluded error values at error correction of acquired inhomogeneity after correction are within $\pm 18.5 \mu \mathrm{V}$ or $\pm 0.46^{\circ} \mathrm{C}$ range from the mean. Estimation of non-excluded error $90 \%$ confidence interval is $\pm 12 \mu \mathrm{V}$ or $\pm 0.3^{\circ} \mathrm{C}$.

\section{CONCLUSIONS}

The following conclusions from the abovementioned results of investigations of the method proposed in [17, 19] acquired during operation time inhomogeneity of thermocouple correction may be done:

1. Proposed method of inhomogeneity of thermocouple correction acquired during operation time provides correction for existing individual drift irregularity of separate segments;

2. The simplest and the best developed NN - three-layer perceptron for proposed method errors investigation was used. Such NN allows correction at considerably less amount of verifications (four times less);

3. The minimum amount of required verifications is seven. Such number is chosen due to the following reasons:

- $\quad$ constrains of the proposed method;

- $\quad$ properties of the three-layer perceptron (according to [21] its training stops if training vector set is too small);

4. The proposed method allows considerable precision increase for existing individual drift irregularity of separate segments;

5. Distribution of nonexclusive error of error correction acquired during operation time inhomogeneity of thermocouples for existing individual drift irregularity of separate segments is close to normal distribution.

6. Maximum nonexclusive error of correction from individual drift irregularity of segments of thermoelectrodes up to $15 \%$ of maximum drift does not exceed $0.46{ }^{\circ} \mathrm{C}$ for $5 \%$ level of significance.

7. The amount of big deviations of correction error from $\mathrm{NN}$ forecast for individual drift irregularity of segments of thermoelectrode greater than $15 \%$ increases considerably; 
8. It is correct to state that the proposed method decreases error due to inhomogeneity 3-6 times in most cases.

9. Big deviations of forecast from real values of error due to inhomogeneity are quite rare. They appear due to simple NN used for investigations in this paper. More complex NN may provide better forecast of error due to inhomogeneity acquired during operation time.

\section{REFERENCES}

[1] Park, R.M. (1993). Manual on the use of thermocouples in temperature measurement (4th ed.). Revision of ASTM special technical publication 470B.

[2] Webster, J.G. (ed.) (1999). The Measurement, Instrumentation, and Sensors: Handbook. Springer.

[3] State Standards of Ukraine. (1994). Thermocouples. Nominal conversional characteristics. DSTU 2837-94.

[4] International Electrotechnical Commission. Thermocouples. Part 2: Tolerances. International standard IEC 584-2.

[5] Sachenko, A. (1988). Accuracy increasing methods development and precision measurement system design for industry. Dissertation abstract for the Doctor of engineering degree. Leningrad: LETI.

[6] Angkawisittpan, N., Manasri, T. (2012). Determination of sugar content in sugar solutions using interdigital capacitor sensor. Measurement Science Review, 12 (1), 8-13.

[7] Li, Z., Sun, Z. (2013). Development of the vortex mass flowmeter with wall pressure measurement. Measurement Science Review, 13 (1), 20-24.

[8] Sachenko, A., Tverdyj, E. (1983). Improving the Temperature Measurement Methods. Kiev: Tehnika.

[9] Kortvelyessy, L. (1981). Thermoelement Praxis. Vulkan-Verlag.

[10] Berezky, O. (1996). Temperature measurement systems that involve artificial intelligence. Abstract of Ph.D. dissertation, Ivan Franko National University of Lviv, Ukraine.

[11] Turchenko, V. (2001). Neural network-based methods and means for improving the effectiveness of distributed sensor data acquisition and processing networks. Abstract of Ph.D. dissertation, Ivan Franko National University of Lviv, Ukraine.

[12] Belousov, I. (1991). Accuracy improvement of multichannel measurement devices based on thermocouples. Ph.D Thesis, Physics and Mechanics Institute of National Academy of Science of Ukraine, Lviv.

[13] Kyrenkov, I. (1976). Some laws of the thermoelectric heterogeneities. Research in the field of temperature measurements. Moscow: VNIIM, 11-15.
[14] Pavlov, B. (1979). Thermoelectric inhomogeneity of thermocouples' electrodes. Moscow: Print House of Standards.

[15] Sloneker, K.C. (2009). Thermocouple inhomogeneity. Ceramic Industry, 159 (4), 13-18.

[16] Vasylkiv, N., Kochan, O. (2010). Investigations of the temperature field profile change on error of inhomogeneous thermocouples. Bulletin of the Ternopil State Technical University, 2, 146-154.

[17] Isotermal Technology Ltd. (1999). Temperature Calibration with Isotech Block Baths.

[18] Vasylkiv, N. (2010). Increasing of the accuracy of temperature measurement using thermocouples in operating process. Abstract of Ph.D. dissertation, Ivan Franko National University of Lviv, Ukraine.

[19] Rogelberg, N., Nuzhnov, A., Pokrovskaya, G. et al. (1969). The stability of the chromel-alumel thermocouples' thermoelectric power at temperatures up to $1200^{\circ} \mathrm{C}$. Investigation of alloys for thermocouples. In Giprotsvetmetobrabotka. Proceedings.

[20] Milchenko, V. (1984). Investigation of the methods and development of the devices for testing the thermocouples based on base metals. Abstract of Ph.D. dissertation, VNIIFTRI, Moscow.

[21] Chyrka, M. (1997). Increasing of the accuracy of temperature measurement using thermocouples in irregular temperature fields. Abstract of Ph.D. dissertation, Ivan Franko National University of Lviv, Ukraine.

[22] Chyrka, M., Vasylkiv, N., Kochan, R. (1999). Modeling of accuracy increase prediction the nonstability of thermocouples conversion characteristic. Bulletin of Ternopil Academy of National Economy, 2, 26-31.

[23] Vasylkiv, N., Kochan, O., Kochan, V. Sachenko, A. (2009). Research of the temperature measurement error from the acquired thermoelectric heterogeneity of thermocouple electrodes. Measuring Equipment and Metrology, 70, 110-117.

[24] Vasylkiv, N., Kochan, O., Kochan, V. (2009). The method of heterogeneity thermocouple error correction. Ukraine Patent no. 92192.

[25] Kohan, O., Kohan, R. Thermocouple sensor. Ukraine Patent no. 97464.

[26] Kröse, B., van der Smagt, P. (1996). An Introduction to Neural Networks. University of Amsterdam.

Received April 26, 2013. Accepted February 3, 2014. 